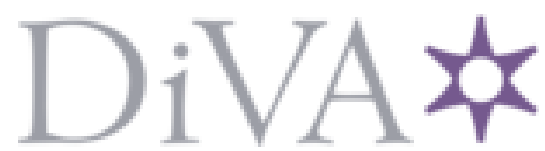

http://www.diva-portal.org

\title{
Preprint
}

This is the submitted version of a paper published in Carbon.

Citation for the original published paper (version of record):

Hinnemo, M., Ahlberg, P., Hägglund, C., Ren, W., Cheng, H-M. et al. (2016)

Scalable residue-free graphene for surface-enhanced Raman scattering.

Carbon, 98: 567-571

http://dx.doi.org/10.1016/j.carbon.2015.11.043

Access to the published version may require subscription.

N.B. When citing this work, cite the original published paper.

Permanent link to this version:

http://urn.kb.se/resolve?urn=urn:nbn:se:uu:diva-269192 


\title{
Scalable Residue-Free Graphene for Surface-
}

\section{Enhanced Raman Scattering}

Malkolm Hinnemo ${ }^{1 \neq}$, Patrik Ahlberg ${ }^{1+}$, Carl Hägglund ${ }^{1}$, Wencai Ren ${ }^{2}$,Hui-Ming Cheng ${ }^{2}$, Shi-Li

Zhang $^{1}$, Zhi-Bin Zhang ${ }^{1 *}$

${ }^{1}$ Solid State Electronics, Department of Engineering Sciences, The Ångström Laboratory, Uppsala University, 75121 Uppsala, Sweden

${ }^{2}$ Shenyang National Laboratory for Materials Science, Institute of Metal Research, Chinese Academy of Sciences, Shenyang 110016, China

$\$$ These authors contributed equally.

\begin{abstract}
A room-temperature polymer-assisted transfer process is developed for large-area, single-layer graphene grown by means of chemical vapor deposition (CVD). This process leads to transferred graphene layers free of polymer contamination. The absence of polymer residues boosts the surface-enhanced Raman scattering (SERS) of the CVD graphene with gold nanoparticles (Au NPs) deposited atop by evaporation. The SERS enhancement of the CVD graphene reaches $\sim 120$ for the characteristic 2D peak of graphene, the highest enhancement factor achieved to date, when the Au NPs are at the threshold of percolation. Our simulation supported by experiment suggests that the polymer residues persistently present on the graphene transferred by the conventional polymer-assisted method are equivalent to an ultrathin film of less than $1 \mathrm{~nm}$ thickness. The presence of polymer residues drastically reduces SERS due to the separation of the Au NPs from the underlying graphene. The scalability of CVD graphene opens up for the possibility of graphene-based SERS sensors.
\end{abstract}

\footnotetext{
* Corresponding author. E-mail: zhibin.zhang@ angstrom.uu.se (Zhi-Bin Zhang)
} 
1. Introduction: Raman scattering is a powerful method to reveal a material's fingerprint information including that of carbon materials such as graphite,[1] carbon nanotubes,[2] fullerenes,[3] and recently graphene.[4] It can be used for identification of defects, phases, molecule and more. Raman scattering however suffers from rather weak signals due to the limited Raman cross-section of common analytes. To break this bottleneck, surface enhanced Raman scattering (SERS) has proven useful. This phenomenon originates from the occurrence of surface plasmon resonances (SPR) in a metal nanostructure, especially those of $\mathrm{Au}$ or $\mathrm{Ag}$, induced by incident light. High electromagnetic field in the vicinity of the metal nanostructures ("hot spots") is created via SPR and strongly enhances the Raman scattering. In this way, SERS enables increased sensitivity even to the level required for single molecule detection.[5] Although SERS opens up for use of Raman spectroscopy for applications that were previously not practically possible,[6,7] it has not yet reached widespread application, mainly due to the technological challenges associated with the requirement of sensitivity, selectivity, uniformity, and reproducibility.

Graphene has since it was first exfoliated mechanically[8] sparked great interest among research and industrial communities due to its various exceptional properties.[9] With its atomic thickness, perfect honeycomb structure, delocalized pi-bonds and its lack of band gap making all wavelengths Raman resonant,[10] single-layer graphene (SLG) has great potential for advancing the SERS technology.[11] It has been used as a Raman probe for in-depth SERS studies and as a flat uniform substrate for a reproducible SERS response.[12,13] The two most intense peaks of the Raman spectrum of SLG are the G peak at $\sim 1580 \mathrm{~cm}^{-1}$ and the $2 \mathrm{D}$ peak at $\sim 2700 \mathrm{~cm}^{-1}$ with the latter being the most characteristic for graphene. With gold nanoparticles (Au NPs) deposited by evaporation, a SERS enhancement factor of up to 120 has recently been reported for the G peak on a clean SLG obtained by mechanical cleavage of graphite.[14] For SLG grown by chemical vapor deposition (CVD) that is transferred onto oxidized $\mathrm{Si}$, a much lower enhancement factor, of around 50, was obtained.[15] In general, CVD graphene has to be transferred from the growth substrate (e.g., $\mathrm{Cu}$ ) onto other substrates for research and applications. In a conventional polymer-assisted transfer process, a polymer layer, most frequently poly methyl methacrylate (PMMA) is employed as support.[16-19] For a successful transfer, samples are usually baked at a temperature slightly higher than the glass transition 
temperature of PMMA. However, this step causes degradation of the PMMA [20] that leads to persistent PMMA residues on graphene.[21] Because of the detrimental effects on various properties, great effort has been made to reduce the PMMA residues.[22,23] Alternative polymers like polycarbonate have also been used that leave less residues but suffer from more wrinkles after transfer.[24] Graphene grown on a $\mathrm{Cu}$ foil can be directly transferred to a desired substrate without using intermediate polymer support, but this technique is applicable to limited types of substrates.[25,26] In addition, transfer of graphene with negligible residue can also be realized by using adhesive tapes.[27]

In this report, we demonstrate a room-temperature PMMA-assisted transfer process that has led to a clean surface for the CVD graphene. The cleanliness results in a significantly enhanced SERS performance of graphene decorated with Au NPs. This allows us to systematically study the SERS of CVD graphene and the effect of PMMA residues which have not been well investigated yet. We show that the difference between the reported SERS-values of mechanically cleaved graphene and CVD graphene is due to polymer residues from the conventional transfer process.

2. Methods: The SLGs were grown by means of thermal CVD on Cu foil (Alfa Aesar) with methane as the carbon source. As shown in Figure 1(a), PMMA is coated on the SLGs as support for the transfer of the SLGs. The SLGs were separated from the $\mathrm{Cu}$ foils by $\mathrm{FeCl}_{3}$ etching and moved onto the oxidized $\mathrm{Si}$ substrate. In the conventional process (hereafter referred to as "Conv-transfer"), baking at $150{ }^{\circ} \mathrm{C}$ on a hotplate under atmospheric conditions for 30 min was performed in order to remove trapped water and improve the adhesion of the SLGs to the oxidized Si. In the room temperature process (hereafter referred to as "RT-transfer"), we modified the conventional one by treating the SLGs at room temperature and low pressure ( 0.02 MPa). Removal of PMMA by acetone completed the transfer processes. Gold with different nominal thicknesses was deposited by resistive evaporation.

Characterizations: The presence of PMMA residues was evaluated by X-ray Photoelectron Spectroscopy (XPS) and atomic force microscopy (AFM). XPS measurements were made at 8 spots on each sample and the results were averaged. The morphology of Au NPs was characterized by means of Scanning Electron Microscopy (SEM). Raman scattering was measured on a Renishaw "inVia" Raman Spectrometer with a $20 \times$ lens. On each sample, 
measurements at 9 randomly selected spots were made and averaged. The enhancement factor is defined as the ratio of the peak area, integrated over wavelength, of G and 2D band with Au NPs to that without.

Simulation: Three dimensional model calculations were carried out by means of finite element methods (FEM) using COMSOL Multiphysics. In the model, a periodic array of horizontally oriented rounded cylinders was used to represent the distribution of Au NPs with $8 \mathrm{~nm}$ nominal thickness. Based on SEM images, the cylinders were assumed to be $20 \mathrm{~nm}$ in diameter, $100 \mathrm{~nm}$ in length and with $10 \mathrm{~nm}$ rounding radii at the two ends, and separated by gaps of $6 \mathrm{~nm}$ in both the longitudinal and transversal directions. The optical properties of $\mathrm{Au}$ were modeled with surface scattering contributions taken into account.[28] Under the cylinders, PMMA, SLG, 300 $\mathrm{nm} \mathrm{SiO}_{2}$ and $\mathrm{Si}$ were stacked. The graphene was modeled as a $0.34 \mathrm{~nm}$ thick layer with a fixed $n$ $=3$ and an extinction coefficient varying according to $k=1.82 \times \lambda$ where $\lambda$ is the wavelength in $\mu \mathrm{m}$.[29] A tetrahedral mesh with $1.2 \times 10^{5}$ elements and a maximum mesh size of $8 \mathrm{~nm}$ was employed. In critical regions, such as near the edge formed by the intersection of the particle and substrate surfaces, the mesh size was reduced down to $0.5 \mathrm{~nm}$. Further refinement does not reduce the error significantly. 

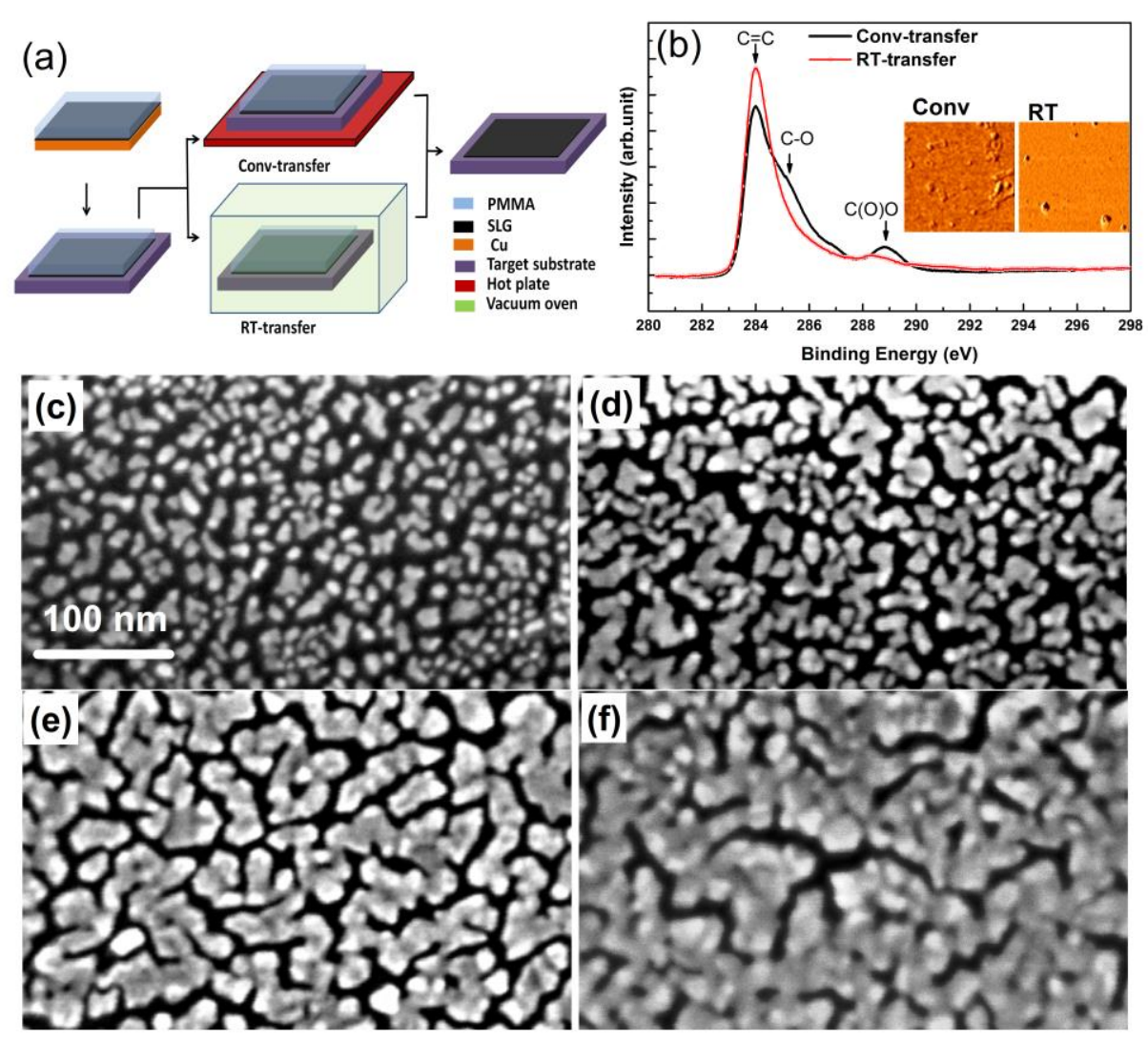

Figure 1. A sketch of Conv- and RT-transfer of CVD-grown SLG (a); XPS and AFM images (inset) obtained from a Conv-transferred SLG (solid line) and an RT-transferred one (solid line with dots) (b); SEM images of Au NPs on RT-transferred SLGs with 2 (c), 4(d), 8(e) and $12 \mathrm{~nm}$ (f) nominal thickness of Au.

3. Results and Discussion: The XPS spectra shown in Figure 1(b) display a substantial difference between the two transfer processes. While the Conv-transferred SLGs show both an appreciable carboxyl group [C(O)O] peak near $289 \mathrm{eV}$ and a carbon-oxygen (C-O) peak at 286 $\mathrm{eV}$, both signatures of polymer residues, $[30,31]$ and a reduced $\mathrm{sp}^{2} \mathrm{C}=\mathrm{C}$ signal at $284 \mathrm{eV}$, the RTtransferred ones provide a diminished $\mathrm{C}(\mathrm{O}) \mathrm{O}$ signature and an almost invisible $\mathrm{C}-\mathrm{O}$ peak next to a profound $\mathrm{C}=\mathrm{C}$ peak. The result indicates that the RT-transferred SLGs are free from PMMA residues. AFM imaging also shows differences (inset of Figure 1(b)). The PMMA-residue free RT-transferred graphene is characterized by a surface roughness only $0.7 \mathrm{~nm}$ compared to 5.2 $\mathrm{nm}$ for the Conv-transferred counterpart. During baking at elevated temperature, substantial degradation of the PMMA occurs, making the PMMA much more difficult to remove.[32] By means of scanning tunneling spectroscopy, it has been observed that the SLG transferred by the conventional method is covered with a relatively large area ultra-thin layer of PMMA residues 
that is conformal[33] to the graphene surface. Unlike PMMA residues in the form of particles and patches, this layer is generally undiscernible by SEM and AFM imaging.

When Au is deposited by evaporation on the RT-transferred SLGs, Au NPs are spontaneously formed as shown in the SEM images in Figure 1(c) to (f). The size and shape of the Au NPs evolve as the $\mathrm{Au}$ increases in nominal thickness. The Au NPs are almost spherical at $2 \mathrm{~nm}$ (Figure 1(c)) and become more complicated with narrower gaps between the adjacent particles as the nominal thickness increases (Figure 1(d), (e)). When the nominal thickness reaches $8 \mathrm{~nm}$ (Figure 1(e)), the coverage of $\mathrm{Au}$ is close to the percolation threshold as determined by field effect conductance measurements using a liquid top gate when the current on/off ratio starts to decreases. At $12 \mathrm{~nm}$, the $\mathrm{Au}$ is percolated (Figure 1(f)) and the current ratio is almost unity. There is no observable difference in Au morphology when the Conv-transferred SLGs are used.

The SERS of the RT-transferred SLGs obtained by averaging 9 spots on each sample with wavelength of 532, 633 and $785 \mathrm{~nm}$ for excitation are shown in Figure 2(a), (b) and (c), respectively. The SERS enhancement factors for the 532 and $633 \mathrm{~nm}$ excitation are obtained by calculating the ratio of the area of G (or 2D) peak of SLG with Au NPs to that without them (Figure 2(d)). This is unobtainable for the $785 \mathrm{~nm}$ excitation since the Raman peaks are invisible without $\mathrm{Au}$ NPs (Figure 3(c)). Several observations deserve to be mentioned.
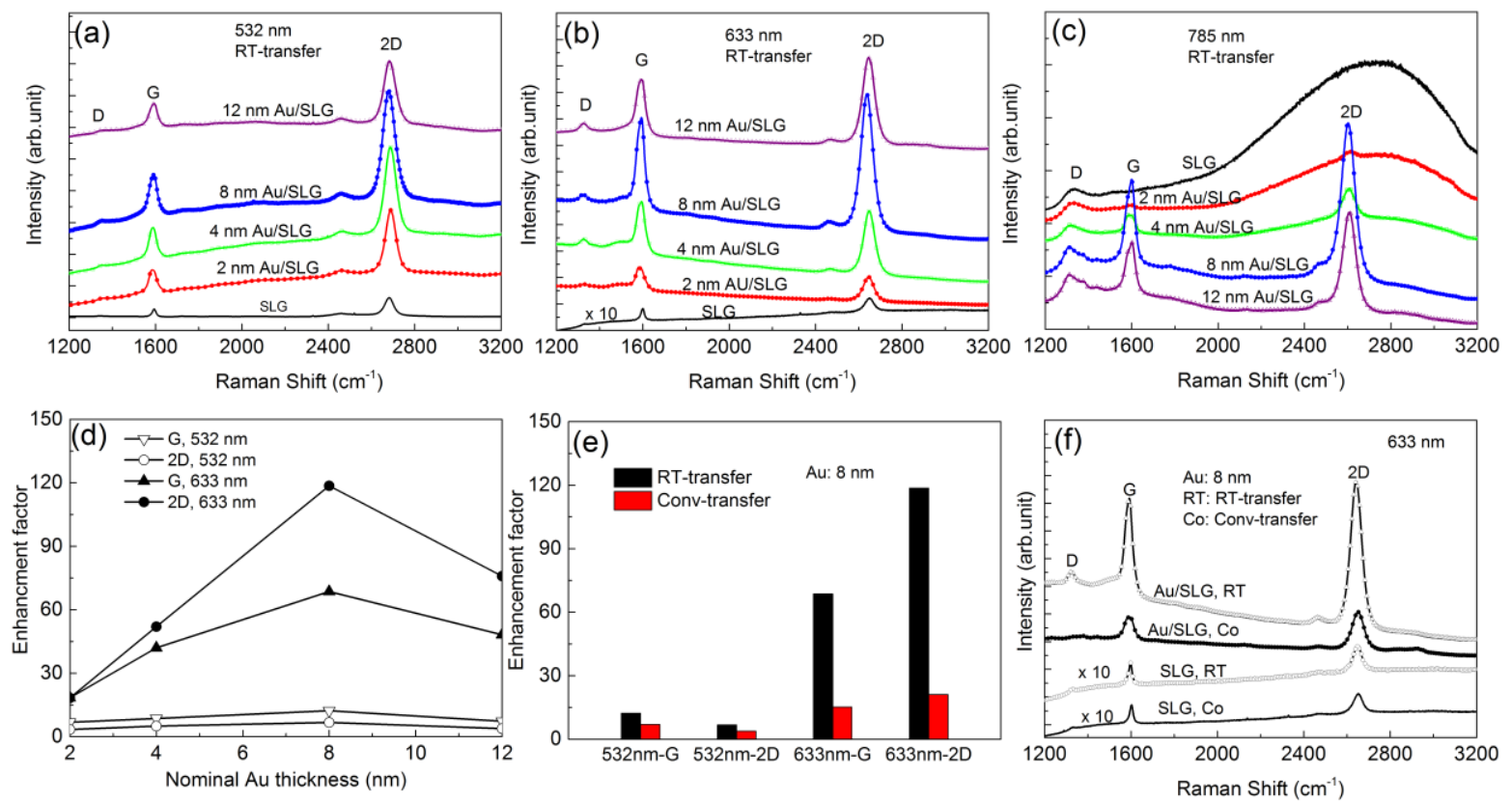

Figure 2. SERS of RT-transferred SLGs coated with Au NPs with various nominal thicknesses, with incident light at 532 (a), 633 (b) and $785 \mathrm{~nm}$ (c); a plot of enhancement factors of G and 2D 
peak as a function of nominal thickness of $\mathrm{Au}$ at 532 and $633 \mathrm{~nm}$ excitation (d); histogram of enhancement factor of the RT- and Conv-transferred SLGs with Au NPs of 8-nm nominal thickness for $\mathrm{G}$ and $2 \mathrm{D}$ peak at 532 and $633 \mathrm{~nm}$ excitation (e); and the comparison of SERS of the RT- and Conv-transferred SLGs at $633 \mathrm{~nm}$ excitation (f).

First, for the 532 and $633 \mathrm{~nm}$ excitations, the SERS enhancement factors of the $\mathrm{G}$ and the 2D bands increase as the nominal thickness of Au increases up to $8 \mathrm{~nm}$. At this thickness, the SERS enhancement starts to decrease, which is consistent with a previous report.[34] This can be understood by the structure of the deposited Au layer. Since the SERS enhancement scales with the electric field strength $(E)$ in proportion to $E^{4},[35]$ it is especially pronounced in small gaps between adjacent particles, where mutual field enhancements can result in hot spots with very high local field strength. Second, the SERS enhancement is more profound for the G band than the $2 \mathrm{D}$ band at the $532 \mathrm{~nm}$ wavelength for all film thicknesses. Conversely, it is much stronger for the 2D band than the $\mathrm{G}$ band at the $633 \mathrm{~nm}$ excitation (Figure 2(d)). Third, at $785 \mathrm{~nm}$, a large bump ranging from 2000 to $3000 \mathrm{~cm}^{-1}$ is observed. This is assigned to the $\mathrm{SiO}_{2} / \mathrm{Si}$ substrate as it is also observed in the absence of SLG and Au NPs. The presence of $\mathrm{Au}$ NPs effectively suppresses the bump and at the same time makes the Raman bands of SLG appear. Finally, the SERS enhancement factor for the RT-transferred SLGs is much larger than that of the Convtransferred SLGs (Figure 2(e) and (f)). With the Au NPs of $8 \mathrm{~nm}$ nominal thickness, the SERS enhancement factor is 21 for the 2D peak of the Conv-transferred SLG at $633 \mathrm{~nm}$ excitation, which is in line with previous reports.[36] The RT-transferred SLG on the other hand achieves an enhancement factor of $\sim 120$ under the same conditions, about 6 times larger than that of the Conv-transferred one. This is the highest enhancement factor of the 2D peak reported for both mechanically cleaved and CVD-grown SLGs. When the PMMA residues exist as an ultrathin layer on the Conv-transferred SLG, it separates the Au NPs from the underlying SLG. Any increase in this physical distance leads to a substantial reduction in the SERS enhancement. This is confirmed by using a $12-\mathrm{nm}$ thick $\mathrm{Al}_{2} \mathrm{O}_{3}$ film as a spacer between a RT-transferred SLG and the $8 \mathrm{~nm}$ Au NPs (result not shown), which reduces the enhancement factor for the 2D band from $\sim 120$ to 3 at the $633-\mathrm{nm}$ excitation.

To shed light on the influence of PMMA residues on the SERS enhancement of SLG, electromagnetic FEM are employed. The Au NPs of 8-nm nominal thickness is modeled as an array of rounded $\mathrm{Au}$ cylinders as shown in Figure 3(a). 

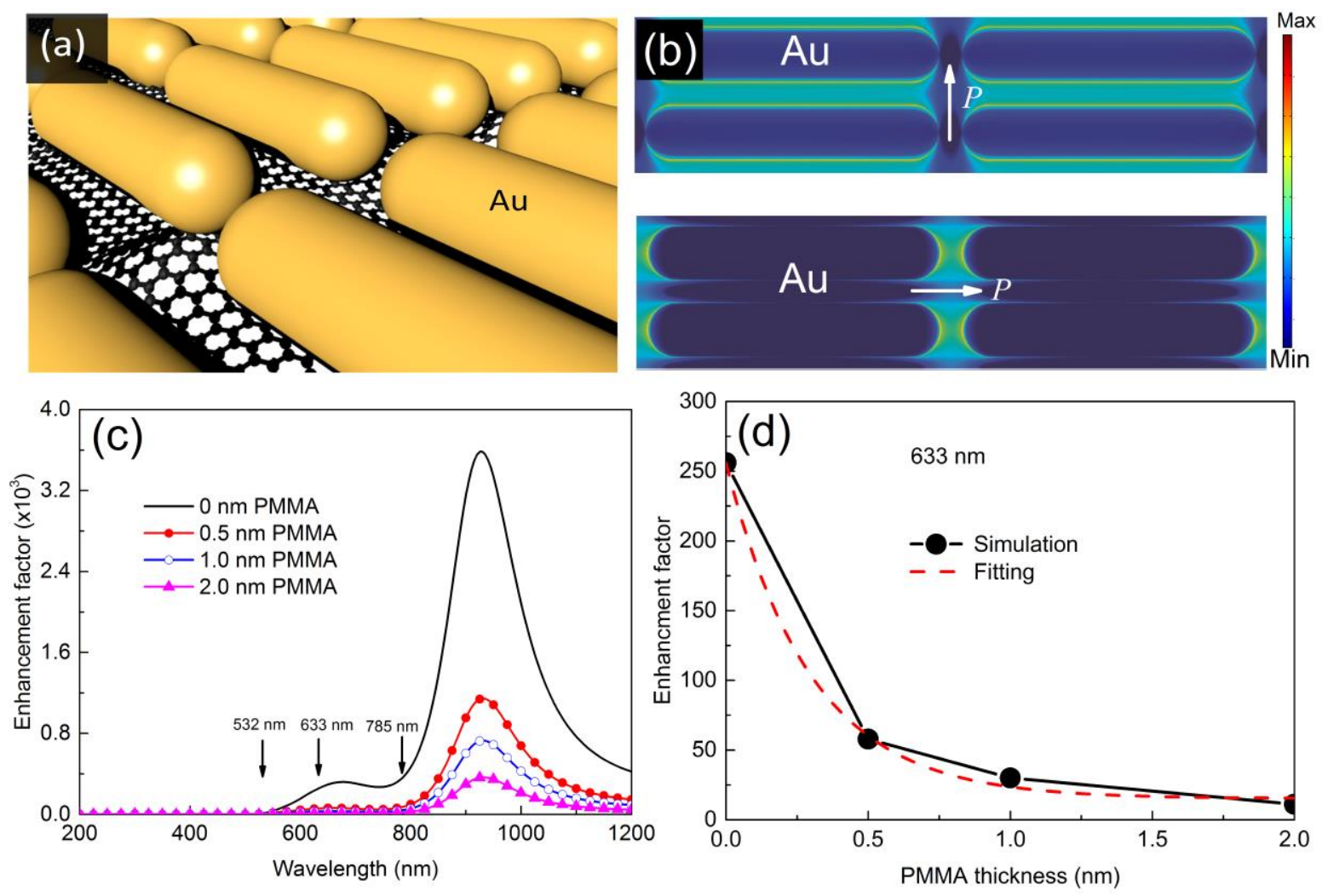

Figure 3. A 3-dimentional sketch of the model used for simulation where the cylinders represent the distribution of the Au NPs with $8 \mathrm{~nm}$ of nominal thickness (a); a snapshot of electromagnetic field distribution under the irradiation of the incident light of $672 \mathrm{~nm}$ wavelength with the polarization oriented along the short axis (Upper part of (b)) and of $928 \mathrm{~nm}$ wavelength with polarization oriented along the long axis of the cylinders (lower part of (b)), respectively, where the "P" represents the polarization direction; the average enhancement factor as a function of wavelength with different PMMA thickness between the cylinders and the underlying SLG (c), the dependence of enhancement factor at $633 \mathrm{~nm}$ on the PMMA thickness (d).

The cylinders are distanced by $6 \mathrm{~nm}$ from each other. With the light-induced SPR, electromagnetic hot spots are formed as depicted in the snapshots of the electromagnetic field energy when the polarization of the light is along the short (upper part of Figure 3(b)) and long (lower part of Figure 3(b)) axis of the cylinders, respectively. Hot spots locate in the vicinity of the $\mathrm{Au}$ edges perpendicular to the polarization of the incident light and the field intensity decays rapidly away from the edges. An appreciable mutual field enhancement from the adjacent cylinders is observed. By averaging over the cylinders with their long axis parallel and perpendicular to the polarization direction of the incident light, the average enhancement factor 
as a function of wavelength of the incident light is plotted with different PMMA interlayer thickness as shown in Figure 3(c). The enhancement factor from the simulation is 11, 256 and 361 for the clean SLG with the excitation wavelength at 532, 633 and $785 \mathrm{~nm}$, respectively. The influence of the presence of PMMA interlayer on the average enhancement factor at $633 \mathrm{~nm}$ excitation is shown in Figure 3(d). It is found that the SERS enhancement decreases exponentially as the PMMA increases in thickness. When the PMMA thickness is $\sim 0.6 \mathrm{~nm}$, the SERS enhancement is reduced by around $\times 6$. This is consistent with the experimental result that the SERS enhancement of the Conv-transferred SLGs is $\times 6$ lower than the RT-transferred ones at $633 \mathrm{~nm}$ (Figure 2(e)). Therefore, the combination of our simulation and experimental results indicates that PMMA residues are present in the form of ultra-thin layer that is conformal to the underlying graphene in addition to the form of particles and patches as shown in the AFM image (inset of Figure 1(b)) on the Conv-transferred graphene. The thickness of the PMMA layer is below $1 \mathrm{~nm}$. The substantial influence of PMMA residues points out the importance of surface cleanliness of graphene for SERS application. It is noted that the influences associated with the grain boundaries, variation of particle sizes, defects or height variation of the graphene are not taken into account during the simulation.

4. Conclusion: In summary, a room-temperature transfer process leading to the CVD graphene free of PMMA contamination is shown. The residue-free CVD-graphene is found to boost the SERS effect with Au NPs and it renders a maximum enhancement by about 120 times for the 2D peak, the highest for CVD-graphene to date. Simulation combined with experiments suggests that the polymer residues are equivalent to a thin film with thickness below $1 \mathrm{~nm}$ and reduce the SERS effect drastically. Our room-temperature polymer-assisted transfer process leads to highquality transfer of CVD-grown graphene free of polymer residues and ensures the highest SERS effect. The scalability of CVD-grown graphene opens up for the possibility of graphene-based SERS sensors.

5. Acknowledgement: We would like to thank Mr. Jin Dai for SEM measurement, Dr. Subimal Majee for AFM imaging, and Dr. Min Yan and Dr. Shun Yu, for fruitful discussions. The study is supported by the Knut and Alice Wallenberg Foundation (2011.0113 and 
2011.0082), by the Swedish Foundation of Strategic Research (Dnr SE13-0061) and by the Swedish Research Council (VR, No.621-2014-5591).

\section{References}

[1] Tuinstra F, Koenig JL. Raman Spectrum of Graphite. J Chem Phys 1970;53:1126. doi:10.1063/1.1674108.

[2] Dresselhaus MS, Dresselhaus G, Saito R, Jorio A. Raman spectroscopy of carbon nanotubes. Phys Rep 2005;409:47-99. doi:10.1016/j.physrep.2004.10.006.

[3] Dresselhaus MS, Dresselhaus G, Eklund PC. Raman Scattering in Fullerenes. J Raman Spectrosc 1996;27:351-71.

[4] Ferrari AC, Meyer JC, Scardaci V, Casiraghi C, Lazzeri M, Mauri F, et al. Raman Spectrum of Graphene and Graphene Layers. Phys Rev Lett 2006;97:1-4. doi:10.1103/PhysRevLett.97.187401.

[5] Kneipp K, Wang Y, Kneipp H, Perelman L, Itzkan I, Dasari R, et al. Single Molecule Detection Using Surface-Enhanced Raman Scattering (SERS). Phys Rev Lett 1997;78:1667-70. doi:10.1103/PhysRevLett.78.1667.

[6] Sharma B, Frontiera RR, Henry A-I, Ringe E, Van Duyne RP. SERS: Materials, applications, and the future. Mater Today 2012;15:16-25. doi:10.1016/S13697021(12)70017-2.

[7] Altun AO, Youn SK, Yazdani N, Bond T, Park HG. Metal-dielectric-CNT nanowires for femtomolar chemical detection by surface enhanced Raman spectroscopy. Adv Mater 2013;25:4431-6. doi:10.1002/adma.201300571.

[8] Novoselov KS, Geim AK, Morozov S V, Jiang D, Zhang Y, Dubonos S V, et al. Electric field effect in atomically thin carbon films. Science 2004;306:666-9.

doi:10.1126/science.1102896.

[9] Geim AK, Novoselov KS. The rise of graphene. Nat Mater 2007;6:183-91. doi:10.1038/nmat 1849 .

[10] Ferrari AC, Basko DM. Raman spectroscopy as a versatile tool for studying the properties of graphene. Nat Nanotechnol 2013;8:235-46. doi:10.1038/nnano.2013.46.

[11] Xu W, Mao N, Zhang J. Graphene: A platform for surface-enhanced Raman spectroscopy. Small 2013;9:1206-24. doi:10.1002/smll.201203097. 
[12] Schedin F, Lidorikis E, Lombardo a, Kravets VG, Grigorenko a N, Novoselov KS, et al. Surface Enhanced Raman Spectroscopy of Graphene. Scan Electron Microsc 2010:1-11. doi:10.1021/nn1010842.

[13] Ling X, Xie L, Fang Y, Xu H, Zhang H, Kong J, et al. Can graphene be used as a substrate for Raman enhancement? Nano Lett 2010;10:553-61. doi:10.1021/n1903414x.

[14] Lee J, Shim S, Kim B, Shin HS. Surface-enhanced Raman scattering of single- and fewlayer graphene by the deposition of gold nanoparticles. Chem - A Eur J 2011;17:2381-7. doi:10.1002/chem.201002027.

[15] Kim N, Oh MK, Park S, Kim SK, Hong BH. Effect of gold substrates on the raman spectra of graphene. Bull Korean Chem Soc 2010;31:999-1003.

doi:10.5012/bkcs.2010.31.04.999.

[16] Reina A, Son H, Jiao L, Fan B, Dresselhaus MS, Liu Z, et al. Transferring and identification of single- and few-layer graphene on arbitrary substrates. J Phys Chem C 2008;112:17741-4. doi:10.1021/jp807380s.

[17] Gao L, Ren W, Xu H, Jin L, Wang Z, Ma T, et al. Repeated growth and bubbling transfer of graphene with millimetre-size single-crystal grains using platinum. Nat Commun 2012;3:699. doi:10.1038/ncomms1702.

[18] De La Rosa CJL, Sun J, Lindvall N, Cole MT, Nam Y, Löffler M, et al. Frame assisted $\mathrm{H} 2 \mathrm{O}$ electrolysis induced $\mathrm{H} 2$ bubbling transfer of large area graphene grown by chemical vapor deposition on Cu. Appl Phys Lett 2013;102. doi:10.1063/1.4775583.

[19] Liang X, Sperling BA, Calizo I, Cheng G, Hacker CA, Zhang Q, et al. Toward clean and crackless transfer of graphene. ACS Nano 2011;5:9144-53. doi:10.1021/nn203377t.

[20] Kashiwagi T, Inaba A, Brown JE, Hatada K, Kitayama T, Masuda E. Effects of weak linkages on the thermal and oxidative degradation of poly(methyl methacrylates). Macromolecules 1986;19:2160-8. doi:10.1021/ma00162a010.

[21] Lin Y-C, Lu C-C, Yeh C-H, Jin C, Suenaga K, Chiu P-W. Graphene annealing: how clean can it be? Nano Lett 2012;12:414-9. doi:10.1021/nl203733r.

[22] Li X, Zhu Y, Cai W, Borysiak M, Han B, Chen D, et al. Transfer of Large-Area Graphene Films for High-Performance Transparent Conductive Electrodes. Nano Lett 2009.

[23] Cheng Z, Zhou Q, Wang C, Li Q, Wang C, Fang Y. Toward Intrinsic Graphene Surfaces : A Systematic Study on Thermal Annealing and Wet-Chemical Treatment of SiO2Supported Graphene Devices. Nano Lett 2011;11:767-71. doi:10.1021/nl103977d. 
[24] Wood JD, Doidge GP, Carrion E a, Koepke JC, Kaitz J a, Datye I, et al. Annealing free, clean graphene transfer using alternative polymer scaffolds. Nanotechnology

2015;26:055302. doi:10.1088/0957-4484/26/5/055302.

[25] Martins LGP, Song Y, Zeng T, Dresselhaus MS, Kong J, Araujo PT. Direct transfer of graphene onto flexible substrates 2013. doi:10.1073/pnas.1306508110//DCSupplemental.www.pnas.org/cgi/doi/10.1073/pnas.1306508110.

[26] Wang DY, Huang IS, Ho PH, Li SS, Yeh YC, Wang DW, et al. Clean-lifting transfer of large-area residual-free graphene films. Adv Mater 2013;25:4521-6. doi:10.1002/adma.201301152.

[27] Kim SJ, Choi T, Lee B, Lee S, Choi K, Park JB, et al. Ultra-Clean Patterned Transfer of Single-Layer Graphene by Recyclable Pressure Sensitive Adhesive Films. Nano Lett 2015:150406130050009. doi:10.1021/acs.nanolett.5b00440.

[28] Hägglund C, Zeltzer G, Ruiz R, Thomann I, Lee HBR, Brongersma ML, et al. Selfassembly based plasmonic arrays tuned by atomic layer deposition for extreme visible light absorption. Nano Lett 2013;13:3352-7. doi:10.1021/n1401641v.

[29] Bruna M, Borini S. Optical constants of graphene layers in the visible range. Appl Phys Lett 2009;94:031901. doi:10.1063/1.3073717.

[30] Han Y, Zhang L, Zhang X, Ruan K, Cui L, Wang Y, et al. Clean surface transfer of graphene films via an effective sandwich method for organic light-emitting diode applications. J Mater Chem C 2014;2:201. doi:10.1039/c3tc31722f.

[31] Lee J, Kim Y, Shin H-J, Lee C, Lee D, Moon C-Y, et al. Clean transfer of graphene and its effect on contact resistance. Appl Phys Lett 2013;103:103104. doi:10.1063/1.4819740.

[32] Evans DF, Wennerström H. The Colloidal Domain: Where Physics, Chemistry and Technology Meet. 1999.

[33] Ishigami M, Chen JH, Cullen WG, Fuhrer MS, Williams ED. Atomic structure of graphene on SiO2. Nano Lett 2007;7:1643-8. doi:10.1021/n1070613a.

[34] Xu W, Ling X, Xiao J, Dresselhaus MS, Kong J, Xu H, et al. Surface enhanced Raman spectroscopy on a flat graphene surface. (Supporting Information). Proc Natl Acad Sci U S A 2012;109:9281-6. doi:10.1073/pnas.1205478109.

[35] Maier SA. Plasmonics: Fundamentals and Applications. 2007.

[36] Hao Q, Wang B, Bossard JA, Kiraly B, Zeng Y, Chiang I-K, et al. Surface-Enhanced Raman Scattering Study on Graphene Coated Metallic Nanostructure Substrates. J Phys Chem C 2012;116:7249-54. doi:10.1021/jp209821g. 